\title{
Reliability Measures of Two Dissimilar Units Parallel System Using Gumbel-Hougaard Family Copula
}

\author{
Garima Chopra \\ University Institute of Engineering and Technology \\ Maharshi Dayanand University, Rohtak, Haryana, India \\ Corresponding Author: garima.chopra@gmail.com \\ Mangey Ram \\ Department of Mathematics; Computer Science and Engineering \\ Graphic Era Deemed to be University, Dehradun, Uttarakhand, India \\ E-mail: drmrswami@yahoo.com
}

(Received August 2, 2018; Accepted November 5, 2018)

\begin{abstract}
The goal of the present study is to investigate the availability and the reliability of the system, which has two dissimilar units in the parallel network under copula. Other key parameters, such as mean time to failure (MTTF) and expected profit are also evaluated. Simultaneous malfunctioning of units, common cause failure and human fault are the causes of system breakdown. The present mathematical model is examined under the assumption that each failure rate is constant and is exponentially distributed. The system undergoes repair in the completely failed state as well as in degraded state. In the case of complete failure, the system is repaired by two repair facilities and that are tackled by utilizing Gumbel-Hougaard family copula. The present system has been studied by applying the concepts of probability theory, supplementary variable technique and Laplace transformation.
\end{abstract}

Keywords- Reliability, Availability, MTTF, Copula.

\section{Introduction}

In the present scenario of the competitive world, increasing complexity in components and systems has made it imperative for industries to produce highly reliable, user friendly, efficient and cost effective systems. Reliability is the probability that a unit or a machine will perform its specified task adequately for an assured period of time under the given set of conditions. Customer satisfaction is highly governed by the reliability, availability and the performance of the system. Thus, reliability analysis is an important phase in the planning, designing and manufacturing of any system. In recent time, it has emerged as one of the most challenging and demanding theory.

In spite of advanced automation techniques, manpower is involved in system operation and hence system may fail at any instant due to unexpected human activity. Lack of training, inadequate experience, mental stress, high noise levels, improper work layouts, inadequate tools and poorly written manuals are the chief causes of human error. Apart from human error, some other major causes of system failure are common cause failure, catastrophic failure, hardware failure, partial failure and unavailability of repairman. Common cause failure occurs when multiple units or components fail in the same manner due to a single cause. Temperature, humidity, pressure, equipment design deficiencies and maintenance errors are some of the reasons of common cause failures. 
International Journal of Mathematical, Engineering and Management Sciences

Vol. 4, No. 1, 116-130, 2019

https://dx.doi.org/10.33889/IJMEMS.2019.4.1-011

Ram (2013) has summarized the survey of various reliability approaches that are used in the fields of engineering and applied sciences. Complex systems are extensively discussed by a number of earlier authors like Dhillon and Yang (1992, 1993), Kontoleon and Kontoleon (1974); Gupta and Agarwal (1984) by considering various failure and repair disciplines. Recently, Saxena et al. (2019) analyzed the performance of the water treatment plant by evaluating its various reliability measures. Zheng et al. (2018) considered component importance analysis of a real-time computing system with warm standby redundancy and common cause failures. Kumar et al. (2017) studied multistate casting system with the help of supplementary variable technique and Laplace transform.

Parallel redundancy is an important commonly used method for enhancing system reliability. Li (2016) discussed the pros and cons of the active redundancy and standby redundancy. The author utilized Markov model technique to evaluate mean time between failures for active and standby redundant systems. Chung (1981) used the Laplace transform technique for analyzing a redundant system comprising of two dissimilar three-state active units and one standby unit. Author has assumed that the units of the system may go down due to two mutually exclusive failure modes or by common cause failure. Dhillon and Viswanath (1991) obtained reliability, steady state availability, time dependent availability and MTTF for three models, where each model corresponds to two distinct units parallel system with common cause failure. Dhillon and Anuda (1993) further considered the same parallel system with gamma distributed repair time. Authors employed the supplementary variable technique to provide the Laplace transform solution of state probability equations. Sridharan and Mohanavadivu (1997) studied three Markov models each representing the parallel system comprising of two non-identical units with different repair rules. In model 1, the system undergoes repair in both the partially failed state as well as in the completely failed state, whereas in model 2 it undergoes repair only in the case of partial failure. The considered system in model 3 is not at all repairable. Researchers concluded that the availability, reliability and the MTTF of parallel system shrinks with the increment in common cause failure rate and human error rate. Chopra and Ram (2017) further evaluated the reliability measures of the dissimilar units parallel system by incorporating waiting time.

Agnihotri and Satsangi (1996) analyzed two distinct parallel units system with priority based repair, inspection and post repair. Gupta et al. (1999) used regenerative point technique for studying the system in which the failure times of two dissimilar operating parallel units are correlated. Kumar et al. (2012) analyzed system having priority unit and non priority unit arranged in a parallel configuration. The priority unit is repairable and non priority unit is not repairable and is replaced after a random period of operation. Authors have supposed that the failure and repair times of the priority unit are correlated random variable having bivariate exponential distribution. Malik et al. (2010); Deswal and Malik (2015) also used regenerative point technique for analyzing parallel systems. EL-Sherbeny (2013) considered four types of failure: hardware failure, common cause failure, critical human error and non-critical human error to analyze the performance of two dissimilar units parallel system under preventive maintenance and two types of repair. They employed graphical evaluation and review technique to derive various reliability measures.

The concept of Copula is studied by many authors including Nelsen (2006). Copulas are defined as multivariate distribution functions with uniform margins over the interval $[0,1]$. The GumbelHougaard family copula is the bivariate distribution given by: 
International Journal of Mathematical, Engineering and Management Sciences

Vol. 4, No. 1, 116-130, 2019

https://dx.doi.org/10.33889/IJMEMS.2019.4.1-011

$$
C_{\theta}\left(u_{1}, u_{2}\right)=e^{\left(-\left(\left(-\log u_{1}\right)^{\theta}+\left(-\log u_{2}\right)^{\theta}\right)^{1 / \theta}\right)} .
$$

Here, $\theta$ lies in the interval $[1, \infty)$ and $\theta$ controls the dependency between $u_{1}$ and $u_{2}$. GumbelHougaard copula belongs to the Archimedean family of copulas. Independence copula and comonotonicity copula are the special cases of Gumbel-Hougaard family copula for $\theta=1$ and $\theta \rightarrow \infty$ respectively. Gumbel-Hougaard family copula is not symmetric and possesses higher dependence at right tails. The work presented in a series of papers of Ram and Singh (2008, 2010), Ram et al. (2013), Singh and Gulati (2014); Ram and Goyal (2018) is based on copula approach. In all these papers, authors have incorporated two types of repair facilities between neighbouring states. The two repair facilities are modelled by using Gumbel-Hougaard family copula.

Ram and Singh (2008) mentioned several reasons for using copula as a tool for modelling dependence. They applied copula to analyze the complex system which consists of two independent repairable subsystems namely 1-out-of-2:F and 1-out-of- $n: F$ in series. Authors studied system under preemptive resume repair policy. They found significant improvement in the reliability of the system by incorporating copula. Ram et al. (2013) considered standby system where repair of main and standby unit follows general distribution, but repair in case of human error is handled with the aid of Gumbel-Hougaard family copula. Singh and Gulati (2014) further adopted copula to study a standby complex system under waiting time discipline. Authors modelled the need of fast repair in the case of complete failure and human error by using Gumbel-Hougaard family copula distribution. Ram and Goyal (2018) presented a novel concept for three state fault tolerant repairable system with two kinds of repair facilities. They have shown that the coverage factor and copula improves the performance of the system.

Redundant parallel systems are unanimously used in computers, power plants, navigation systems, fire stations, aircraft systems, communication systems and many other critical systems. Therefore, this study is carried out to compute the reliability measures of two distinct units parallel system subjected to two kinds of failure under two repair facilities. The two different types of repair are modelled by using Gumbel-Hougaard family copula. Supplementary variable technique and Laplace transformation are used in the present study.

\section{Model Description}

The system has two dissimilar units, 1 and 2 which are arranged in a parallel network. Simultaneous hardware failure of distinct units, common cause failure and human fault leads to total system failure. Normal, degraded and complete failure are the three states of the present redundant system. These states and corresponding transition diagram is presented in Table 1 and Figure 1 respectively. There are two repair facilities namely exponential and general between the completely failed states $\left(S_{3}, S_{4}, S_{5}\right)$ and the normal state $\left(S_{0}\right)$. 
International Journal of Mathematical, Engineering and Management Sciences

Vol. 4, No. 1, 116-130, 2019

https://dx.doi.org/10.33889/IJMEMS.2019.4.1-011

Table 1. States explanation

\begin{tabular}{|c|l|}
\hline State & \multicolumn{1}{|c|}{ Description } \\
\hline $\mathrm{S}_{0}$ & Normal state: Both dissimilar units are functioning properly \\
\hline $\mathrm{S}_{1}$ & Degraded state: Unit 1 has stopped working and is under repair, Unit 2 is functioning normally \\
\hline $\mathrm{S}_{2}$ & Degraded state: Unit 2 has stopped working and is under repair, Unit 1 is operational \\
\hline $\mathrm{S}_{3}$ & Completely failed state: System failure because of hardware breakdown of both dissimilar units \\
\hline $\mathrm{S}_{4}$ & Completely failed state: System failure due to common cause failure \\
\hline $\mathrm{S}_{5}$ & Completely failed state: System failure due to human error \\
\hline
\end{tabular}

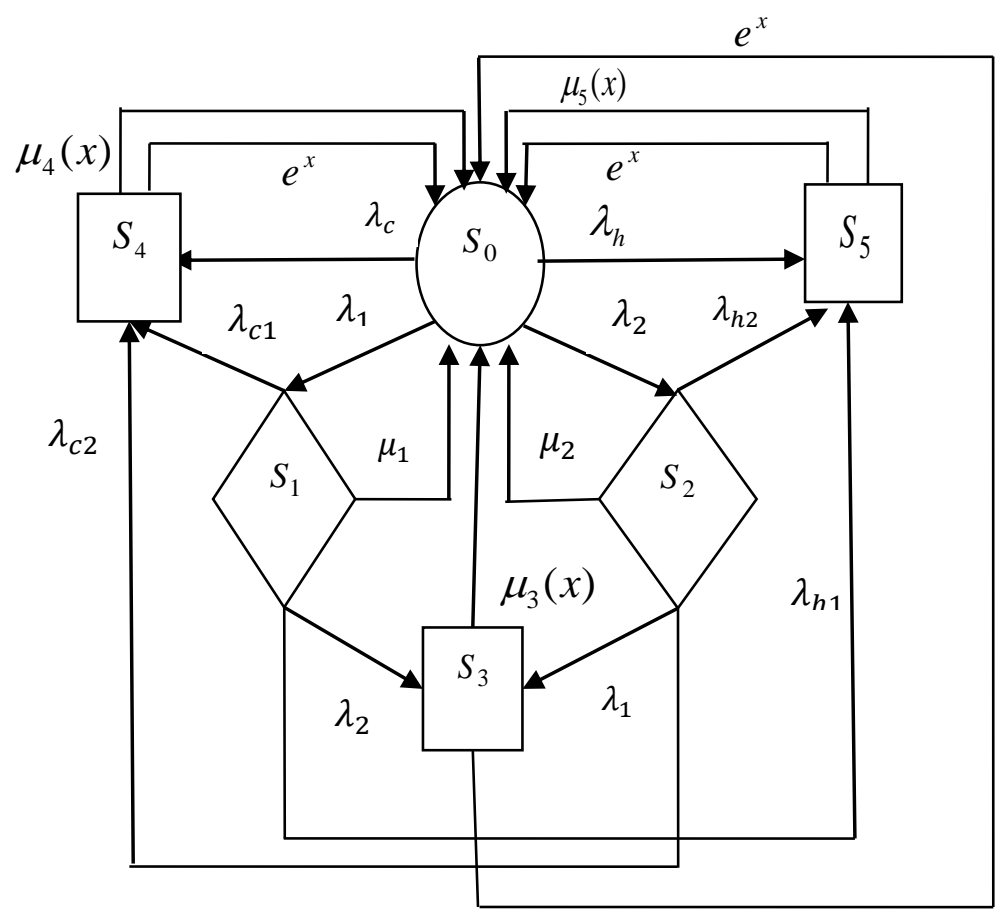

Figure 1. State transition diagram 
International Journal of Mathematical, Engineering and Management Sciences

Vol. 4, No. 1, 116-130, 2019

https://dx.doi.org/10.33889/IJMEMS.2019.4.1-011

\section{Notations and Assumptions}

The notations used in this paper are as follows:

$\begin{array}{ll}t & \text { Time scale } \\ s & \text { Laplace transform variable } \\ S_{i}(i=0,1,2,3,4,5) & \text { Transition states } \\ \lambda_{1} / \lambda_{2} & \text { Hardware failure rate of unit } 1 / \text { unit } 2 \\ \lambda_{c} / \lambda_{c 1} / \lambda_{c 2} & \text { Common cause failure rate for state } S_{0} / S_{1} / S_{2} \\ \lambda_{h} / \lambda_{h 1} / \lambda_{h 2} & \text { The rate of human error for state } S_{0} / S_{1} / S_{2} \\ \mu_{1} / \mu_{2} & \text { Constant repair rate of unit } 1 / \text { unit } 2 \\ \mu_{3}(x) / \mu_{4}(x) / \mu_{5}(x) & \text { Repair rate for the completely failed state } S_{3} / S_{4} / S_{5} \\ P_{i}(t)(i=0,1,2,3,4,5) & \text { Probability of system being in state } S_{i} \text { at instant } t \\ \overline{P_{i}}(s)(i=0,1,2,3,4,5) & \text { Laplace transformation of } P_{i}(t) \\ P_{3}(x, t) / P_{4}(x, t) / P_{5}(x, t) & \text { Probability density function of the system being in completely failed state } S_{3} / S_{4} / S_{5} \text { at instant } t \\ & \text { with elapsed repair time } x \\ C_{\theta 3}\left(u(x), \mu_{3}(x)\right) & \text { Joint probability for repair rate from failed state } S_{3} / S_{4} / S_{5} \text { to normal state } S_{0} \text { according to } \\ / C_{\theta 4}\left(u(x), \mu_{4}(x)\right) & \text { Gumbel-Hougaard copula, where } u(x)=e^{x}, \\ / C_{\theta 5}\left(u(x), \mu_{5}(x)\right) & C_{\theta 3}\left(u(x), \mu_{3}(x)\right)=\text { exp }\left[x^{\theta}+\left(\log \left(\mu_{3}(x)\right)\right)^{\theta}\right]^{1 / \theta} \\ & C_{\theta 4}\left(u(x), \mu_{4}(x)\right)=\text { exp }\left[x^{\theta}+\left(\log \left(\mu_{4}(x)\right)\right)^{\theta}\right]^{1 / \theta} \\ \chi_{3}(s) / \overline{\chi_{4}}(s) / \bar{\chi}(s) & C_{\theta 5}\left(u(x), \mu_{5}(x)\right)=\text { exp }\left[x^{\theta}+\left(\log \left(\mu_{5}(x)\right)\right)^{\theta}\right]^{1 / \theta} \\ \text { Laplace transformation of probability density functions of } \mu_{3}(x) / \mu_{4}(x) / \mu_{5}(x)\end{array}$

In this study, the following assumptions were made:

- The system has two distinct units which are connected in parallel network.

- Initially, the system is in normal state as both parallel units are functional.

- System complete failure occurs on account of common cause failure, human fault and hardware malfunctioning of both units.

- All failures rates are constant. Hardware failure, common cause failure and human failure are statistically independent and exponentially distributed.

- Common cause failure and human error can arise at any instant irrespective of the fact whether one unit is operational $\left(S_{1} / S_{2}\right)$ or both are operational $\left(S_{0}\right)$.

- The system undergoes repair in the completely failed state as well as in degraded state.

- Unit 1 and unit 2 have constant repair rates.

- In complete failed states $\left(S_{3}, S_{4}, S_{5}\right)$, the system is repaired by two repair facilities.

- Gumbel-Hougaard family copula is used for modelling the repairs of completely failed states $\left(S_{3}, S_{4}, S_{5}\right)$.

- After repairing, the system behaves like a new one. 
International Journal of Mathematical, Engineering and Management Sciences

Vol. 4, No. 1, 116-130, 2019

https://dx.doi.org/10.33889/IJMEMS.2019.4.1-011

\section{Analysis and Discussion}

The set of differential equations corresponding to the present model are as follows:

$$
\begin{aligned}
& \begin{aligned}
\left(\frac{\partial}{\partial t}+\lambda_{1}+\lambda_{2}+\right. & \left.\lambda_{h}+\lambda_{c}\right) P_{0}(t)+\mu_{1} P_{1}(t)+\mu_{2} P_{2}(t) \\
& =\int_{0}^{\infty} C_{\theta 3}\left(u(x), \mu_{3}(x)\right) P_{3}(x, t) d x+\int_{0}^{\infty} C_{\theta 4}\left(u(x), \mu_{4}(x)\right) P_{4}(x, t) d x \\
& +\int_{0}^{\infty} C_{\theta 5}\left(u(x), \mu_{5}(x)\right) P_{5}(x, t) d x
\end{aligned} \\
& \left(\frac{\partial}{\partial t}+\lambda_{2}+\lambda_{h 1}+\lambda_{c 1}+\mu_{1}\right) P_{1}(t)=\lambda_{1} P_{0}(t) \\
& \left(\frac{\partial}{\partial t}+\lambda_{1}+\lambda_{h 2}+\lambda_{c 2}+\mu_{2}\right) P_{2}(t)=\lambda_{2} P_{0}(t) \\
& \left(\frac{\partial}{\partial x}+\frac{\partial}{\partial t}+C_{\theta 3}\left(u(x), \mu_{3}(x)\right)\right) P_{3}(x, t)=0 \\
& \left(\frac{\partial}{\partial x}+\frac{\partial}{\partial t}+C_{\theta 4}\left(u(x), \mu_{4}(x)\right)\right) P_{4}(x, t)=0 \\
& \left(\frac{\partial}{\partial x}+\frac{\partial}{\partial t}+C_{\theta 5}\left(u(x), \mu_{5}(x)\right)\right) P_{5}(x, t)=0
\end{aligned}
$$

At instant $t=0$, the probability of the system being in state $S_{i}(i=0,1,2,3,4,5)$ is given by following two equations.

$$
\begin{aligned}
& P_{0}(0)=1 \\
& P_{1}(0)=P_{2}(0)=P_{3}(0)=P_{4}(0)=P_{5}(0)=0
\end{aligned}
$$

The present model is governed by following boundary conditions:

$$
\begin{aligned}
& P_{3}(0, t)=\lambda_{2} P_{1}(t)+\lambda_{1} P_{2}(t) \\
& P_{4}(0, t)=\lambda_{c} P_{0}(t)+\lambda_{c 1} P_{1}(t)+\lambda_{c 2} P_{2}(t) \\
& P_{5}(0, t)=\lambda_{h} P_{0}(t)+\lambda_{h 1} P_{1}(t)+\lambda_{h 2} P_{2}(t)
\end{aligned}
$$

Using Equations (7-11), we obtain Laplace transform solution of Equations (1-6) as follows:

$$
\begin{aligned}
& \overline{P_{0}}(s)=\frac{1}{G(s)} \\
& \overline{P_{1}}(s)=\frac{\lambda_{1}}{\left(s+B_{1}\right)} \overline{P_{0}}(s)
\end{aligned}
$$


International Journal of Mathematical, Engineering and Management Sciences

Vol. 4, No. 1, 116-130, 2019

https://dx.doi.org/10.33889/IJMEMS.2019.4.1-011

$\overline{P_{2}}(s)=\frac{\lambda_{2}}{\left(s+B_{2}\right)} \overline{P_{0}}(s)$

$\overline{P_{3}}(s)=\lambda_{1} \lambda_{2} \overline{P_{0}}(s)\left(\frac{1}{s+B_{1}}+\frac{1}{s+B_{2}}\right)\left(\frac{1-\overline{\chi_{3}}(s)}{s}\right)$

$\overline{P_{4}}(s)=\overline{P_{0}}(s)\left(\lambda_{c}+\frac{\lambda_{1} \lambda_{c 1}}{s+B_{1}}+\frac{\lambda_{2} \lambda_{c 2}}{s+B_{2}}\right)\left(\frac{1-\overline{\chi_{4}}(s)}{s}\right)$

$\overline{P_{5}}(s)=\overline{P_{0}}(s)\left(\lambda_{h}+\frac{\lambda_{1} \lambda_{h 1}}{s+B_{1}}+\frac{\lambda_{2} \lambda_{h 2}}{s+B_{2}}\right)\left(\frac{1-\overline{\chi_{5}}(s)}{s}\right)$

where,

$$
\begin{aligned}
G(s)=s+\lambda_{c}+ & \lambda_{h}+\lambda_{1}+\lambda_{2}-\frac{\lambda_{1} \mu_{1}}{s+B_{1}}-\frac{\lambda_{2} \mu_{2}}{s+B_{2}}-\lambda_{1} \lambda_{2}\left(\frac{1}{s+B_{1}}+\frac{1}{s+B_{2}}\right) \overline{\chi_{3}}(s) \\
& -\left(\lambda_{c}+\frac{\lambda_{c 1} \lambda_{1}}{s+B_{1}}+\frac{\lambda_{c 2} \lambda_{2}}{s+B_{2}}\right) \overline{\chi_{4}}(s) \\
& -\left(\lambda_{h}+\frac{\lambda_{h 1} \lambda_{1}}{s+B_{1}}+\frac{\lambda_{h 2} \lambda_{2}}{s+B_{2}}\right) \overline{\chi_{5}}(s)
\end{aligned}
$$

$B_{1}=\lambda_{2}+\lambda_{c 1}+\lambda_{h 1}+\mu_{1}$ and $B_{2}=\lambda_{1}+\lambda_{c 2}+\lambda_{h 2}+\mu_{2}$

Equation (19) and Equation (20) give Laplace transformation of the probabilities of the system being in up and down state at any moment $t$.

$$
\begin{aligned}
& \overline{P_{u p}}(s)=\overline{P_{0}}(s)+\overline{P_{1}}(s)+\overline{P_{2}}(s)=\frac{1}{G(s)}\left[1+\frac{\lambda_{1}}{\left(s+B_{1}\right)}+\frac{\lambda_{2}}{\left(s+B_{2}\right)}\right] \\
& \overline{P_{\text {down }}}(s)=\overline{P_{3}}(s)+\overline{P_{4}}(s)+\overline{P_{5}}(s)
\end{aligned}
$$

\subsection{Availability}

\subsubsection{System Availability in Comprehensive State}

System availability at any instant $t$ is obtained by setting $\overline{\chi_{3}}(s)=\frac{C_{\theta 3}\left(u(x), \mu_{3}(x)\right)}{s+C_{\theta 3}\left(u(x), \mu_{3}(x)\right)}$,

$\overline{\chi_{4}}(s)=\frac{C_{\theta 4}\left(u(x), \mu_{4}(x)\right)}{s+C_{\theta 4}\left(u(x), \mu_{4}(x)\right)}$ and $\overline{\chi_{5}}(s)=\frac{C_{\theta 5}\left(u(x), \mu_{5}(x)\right)}{s+C_{\theta 5}\left(u(x), \mu_{5}(x)\right)}$ and assuming the values of parameters. In Equation (19), we assumed $\lambda_{1}=0.50, \lambda_{2}=0.40, \lambda_{h}=0.30, \lambda_{h 1}=0.20, \lambda_{h 2}=0.10, \lambda_{c}=$ $0.25, \lambda_{c 1}=0.15, \lambda_{c 2}=0.10, \mu_{1}=1, \mu_{2}=1, \mu_{3}=\mu_{4}=\mu_{5}=1, x=1$ and $\theta=1$ and then applied inverse Laplace transformation. The obtained expression is

$$
\begin{aligned}
A(t)= & 0.183563466447622 e^{(-2.948513192969554 t)} \cos (0.240846721380981 t) \\
& -0.037060362890448 e^{(-2.948513192969554 t)} \sin (0.240846721380981 t) \\
& -0.000095958716709 e^{(-1.721255442519939 t)} \\
& +0.816532492269086
\end{aligned}
$$


International Journal of Mathematical, Engineering and Management Sciences

Vol. 4, No. 1, 116-130, 2019

https://dx.doi.org/10.33889/IJMEMS.2019.4.1-011

\subsubsection{System Availability without Common Cause Failure}

We have computed system availability in this case by considering $\lambda_{1}=0.50, \lambda_{2}=0.40, \lambda_{h}=$ 0.30, $\lambda_{h 1}=0.20, \lambda_{h 2}=0.10, \lambda_{c}=0, \lambda_{c 1}=0, \lambda_{c 2}=0, \mu_{1}=1, \mu_{2}=1, \mu_{3}=\mu_{4}=\mu_{5}=1$, $x=1$ and $\theta=1$ in Equation (19) and after that evaluating inverse Laplace transformation. The obtained system availability at time $t$ is

$$
\begin{aligned}
A(t)= & 0.130506468190394 e^{(-2.759140914229522 t)} \cos (0.450384265458169 t) \\
& +0.133409935834662 e^{(-2.759140914229522 t)} \sin (0.450384265458169 t) \\
& -0.000000000000002 e^{(-1.59999999999999 t)} \\
& +0.869493531809607
\end{aligned}
$$

\subsubsection{System Availability without Human Error}

In this case, system availability is obtained by substituting $\lambda_{1}=0.50, \lambda_{2}=0.40, \lambda_{h}=0$, $\lambda_{h 1}=0, \lambda_{h 2}=0, \lambda_{c}=0.25, \lambda_{c 1}=0.15, \lambda_{c 2}=0.10, \mu_{1}=1, \mu_{2}=1, \mu_{3}=\mu_{4}=\mu_{5}=1$, $x=1$ and $\theta=1$ in Equation (19) and then taking inverse Laplace transformation. We get availability as

$$
\begin{aligned}
A(t)= & 0.119100727810722 e^{(-2.720712511315509 t)} \cos (0.478452459176194 t) \\
& +0.154424764456622 e^{(-2.720712511315509 t)} \sin (0.478452459176194 t) \\
& -0.000097619485088 e^{(-1.576856805828027 t)} \\
& +0.880996891674365
\end{aligned}
$$

Table 2 reveals that initially, there is a sharp decrease in the system availability but in long run it stabilizes to $0.81,0.87$ and 0.88 in the comprehensive state, and in the absence of common cause failure and human error respectively. Figure 2 depicts that system availability is maximum when it is free from human error and is minimum in the comprehensive state. It can also be seen from Figure 2 that system availability in the absence of common cause failure is slightly less as compared to its availability when there is no human error.

\subsection{Reliability}

System reliability is evaluated by equating each repair rate in Equation (19) to zero and then taking inverse Laplace transformation. We have computed system reliability in the following different cases:

\subsubsection{System Reliability in Comprehensive State}

System reliability at any time $t$ is computed by equating each repair rate as zero and considering parameters $\lambda_{1}=0.50, \lambda_{2}=0.40, \lambda_{h}=0.30, \lambda_{h 1}=0.20, \lambda_{h 2}=0.10, \lambda_{c}=0.25$, $\lambda_{c 1}=0.15$ and $\lambda_{c 2}=0.10$ in Equation (19) and then employing inverse Laplace transformation. The attained system reliability is

$$
R(t)=-0.247619047619045 \mathrm{e}^{-1.45 t}+0.714285714285736 \mathrm{e}^{-0.75 t}+0.53 e^{-0.70 t}
$$


International Journal of Mathematical, Engineering and Management Sciences

Vol. 4, No. 1, 116-130, 2019

https://dx.doi.org/10.33889/IJMEMS.2019.4.1-011

\subsubsection{System Reliability without Common Cause Failure}

The expression for system reliability in case of absence of common cause failure is obtained by considering all repairs in Equation (19) as zero and assuming parameters as $\lambda_{1}=0.50, \lambda_{2}=$ $0.40, \lambda_{h}=0.30, \lambda_{h 1}=0.20, \lambda_{h 2}=0.10, \lambda_{c}=0, \lambda_{c 1}=0$ and $\lambda_{c 2}=0$ and then applying inverse Laplace transformation. The evaluated expression is

$R(t)=-0.50 e^{-1.2 t}+1.50 e^{-0.60 t}+0.000000000000002 e^{-0.60 t}$

\subsubsection{System Reliability without Human Error}

Equating each repair rate in Equation (19) to zero and substituting $\lambda_{1}=0.50, \lambda_{2}=0.40$, $\lambda_{h}=0, \lambda_{h 1}=0, \lambda_{h 2}=0, \lambda_{c}=0.25, \lambda_{c 1}=0.15$ and $\lambda_{c 2}=0.10$ followed by inverse Laplace transformation, the attained expression of system reliability is

$R(t)=-0.560606060606044 e^{-1.15 t}+0.727272727272615 e^{-0.60 t}+0.83 e^{-0.55 t}$

Information pertaining to the variation of system reliability over the time when each failure rate has some specific value is shown in Table 2. As depicted in Table 2 and Figure 3, the reliability of the present system shrinks with an increase in time. System reliability is maximum in absence of any chances of human failure. The considered system has the lowest reliability in the comprehensive state. Figure 3 exhibits that in the absence of common cause failure reliability of the assumed system is slightly less as compared to its reliability in absence of human error.

\subsection{MTTF}

MTTF is computed with the help of Laplace transform (Equation 19), by putting each repair rate as zero and evaluating the limit, i.e.,

$M T T F=\lim _{s \rightarrow 0} \overline{P_{u p}}(s)$.

MTTF of the present system is given by

$\operatorname{MTTF}=\frac{1}{\left(\lambda_{1}+\lambda_{2}+\lambda_{c}+\lambda_{h}\right)}\left(1+\frac{\lambda_{1}}{B_{1}}+\frac{\lambda_{2}}{B_{2}}\right)$

The present study examines the impact of each failure rate on MTTF by altering that failure rate as $0.1,0.2,0.3,0.4,0.5,0.6,0.7,0.8$ and 0.9 in Equation (27). The constant values of failure rates are assumed as $\lambda_{1}=0.50, \lambda_{2}=0.40, \lambda_{h}=0.30, \lambda_{h 1}=0.20, \lambda_{h 2}=0.10, \lambda_{c}=0.25$, $\lambda_{c 1}=0.15$ and $\lambda_{c 2}=0.10$ in Equation (27). Table 3 and Figure 4 reveal that, in general, system MTTF decreases as the common cause failure rate, human failure rate and hardware failure rates of both dissimilar parallel units increases. In interval $(0.1,0.2)$, decrease in MTTF with respect to hardware failure rate of unit 1 is high as compared to its decrease with respect to common cause failure rate, human failure rate and hardware failure rate of unit 2. Moreover, Figure 4 exhibits that corresponding to the interval $(0.7,0.9)$, the consequence of the increase of hardware failure rates of unit 1 and unit 2 on system MTTF is the same. The impact of an increase in human failure rate and common cause failure rate on system MTTF is similar. 
International Journal of Mathematical, Engineering and Management Sciences

Vol. 4, No. 1, 116-130, 2019

https://dx.doi.org/10.33889/IJMEMS.2019.4.1-011

\subsection{Cost Analysis}

Expected profit over the time $t$ is evaluated with the help of the following equation

$E_{p}(t)=K_{1} \int_{0}^{t} P_{u p}(t) d t-t K_{2}$

where $K_{1}$ and $K_{2}$ refer to revenue cost and system service cost per unit time, respectively. In a comprehensive state, by using Equation (21) in Equation (28), we obtained the following expression for the system expected profit

$$
\begin{aligned}
E_{p}(t)=K_{1}(- & 0.060823745500503 e^{-2.948513192969554 t} \cos 0.240846721380981 t \\
& +0.17537504224045 e^{-2.948513192969554 t} \sin 0.240846721380981 t \\
& +0.060823745500503 e^{-2.948513192969554 t} \\
& +0.000055749259720 e^{-1.721255442519939 t}+0.816532492269086 t \\
& -0.000055749259720)-t K_{2}
\end{aligned}
$$

We evaluated the expected profit by assuming revenue cost as 1 and changing service cost as $0.10,0.30,0.40$ and 0.70 in Equation (29). Figure 5 indicates that the increasing service cost decreases the expected profit of the present redundant system.

Table 2. Availability and Reliability of system in Comprehensive State

\begin{tabular}{|c|c|c|}
\hline Time & Availability in Comprehensive State & Reliability in Comprehensive State \\
\hline 0 & 1 & 0.544166105 \\
\hline 1 & 0.825396197 & 0.277272269 \\
\hline 2 & 0.816929218 & 0.137399283 \\
\hline 3 & 0.816548245 & 0.067244545 \\
\hline 4 & 0.816532955 & 0.032727808 \\
\hline 5 & 0.816532487 & 0.015891389 \\
\hline 6 & 0.816532489 & 0.007710062 \\
\hline 7 & 0.816532492 & 0.003740462 \\
\hline 8 & 0.816532492 & 0.001815173 \\
\hline 9 & 0.816532492 & 0.000881272 \\
\hline 10 & 0.816532492 & 0.000428092 \\
\hline 11 & 0.816532492 & 0.000208072 \\
\hline 12 & 0.816532492 & 0.000101193 \\
\hline 13 & 0.816532492 & 0.000049243 \\
\hline 14 & 0.816532492 & 0.000023977 \\
\hline 15 & 0.816532492 & \\
\hline
\end{tabular}


International Journal of Mathematical, Engineering and Management Sciences

Vol. 4, No. 1, 116-130, 2019

https://dx.doi.org/10.33889/IJMEMS.2019.4.1-011

Table 3. MTTF as function of failure rates in the Comprehensive State

\begin{tabular}{|c|c|c|c|c|c|c|c|}
\hline$\lambda_{1}$ & $\lambda_{2}$ & $\lambda_{h}$ & $\lambda_{c}$ & $\lambda_{h 1}$ & $\lambda_{h 2}$ & $\lambda_{c 1}$ & $\lambda_{c 2}$ \\
\hline 2.35 & 1.96 & 1.79 & 1.72 & 1.61 & 1.54 & 1.58 & 1.54 \\
\hline 1.97 & 1.76 & 1.66 & 1.60 & 1.54 & 1.49 & 1.51 & 1.49 \\
\hline 1.76 & 1.63 & 1.54 & 1.49 & 1.49 & 1.46 & 1.47 & 1.46 \\
\hline 1.63 & 1.54 & 1.44 & 1.40 & 1.45 & 1.43 & 1.43 & 1.43 \\
\hline 1.54 & 1.49 & 1.36 & 1.32 & 1.41 & 1.40 & 1.40 & 1.40 \\
\hline 1.48 & 1.44 & 1.28 & 1.24 & 1.38 & 1.38 & 1.37 & 1.38 \\
\hline 1.44 & 1.41 & 1.21 & 1.18 & 1.36 & 1.36 & 1.35 & 1.36 \\
\hline 1.41 & 1.39 & 1.15 & 1.12 & 1.34 & 1.35 & 1.33 & 1.35 \\
\hline 1.39 & 1.37 & 1.091 & 1.07 & 1.32 & 1.33 & 1.31 & 1.33 \\
\hline
\end{tabular}

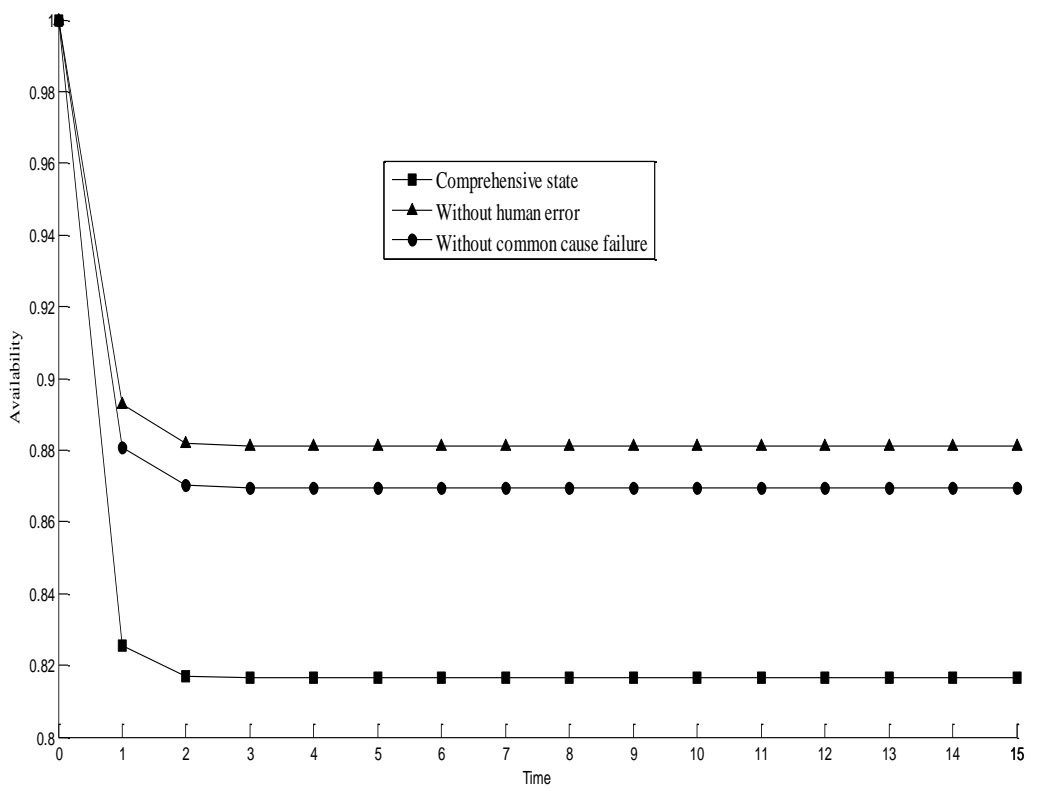

Figure 2. Time vs Availability 
International Journal of Mathematical, Engineering and Management Sciences

Vol. 4, No. 1, 116-130, 2019

https://dx.doi.org/10.33889/IJMEMS.2019.4.1-011

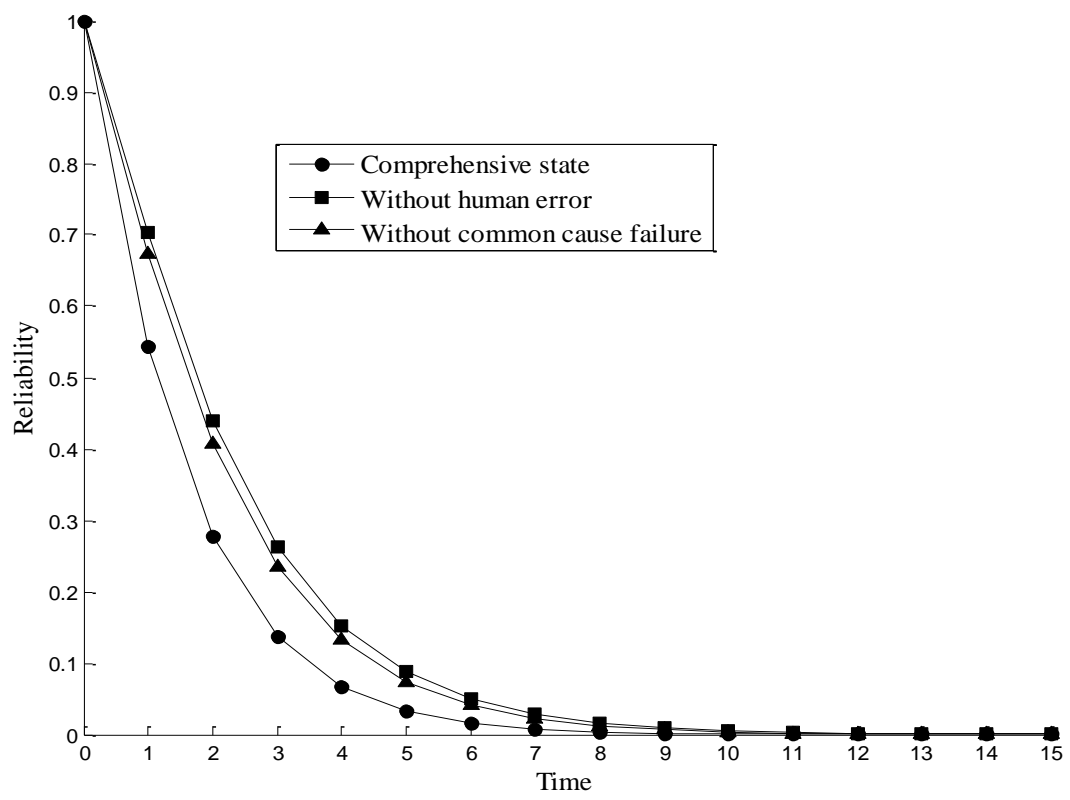

Figure 3. Time vs Reliability

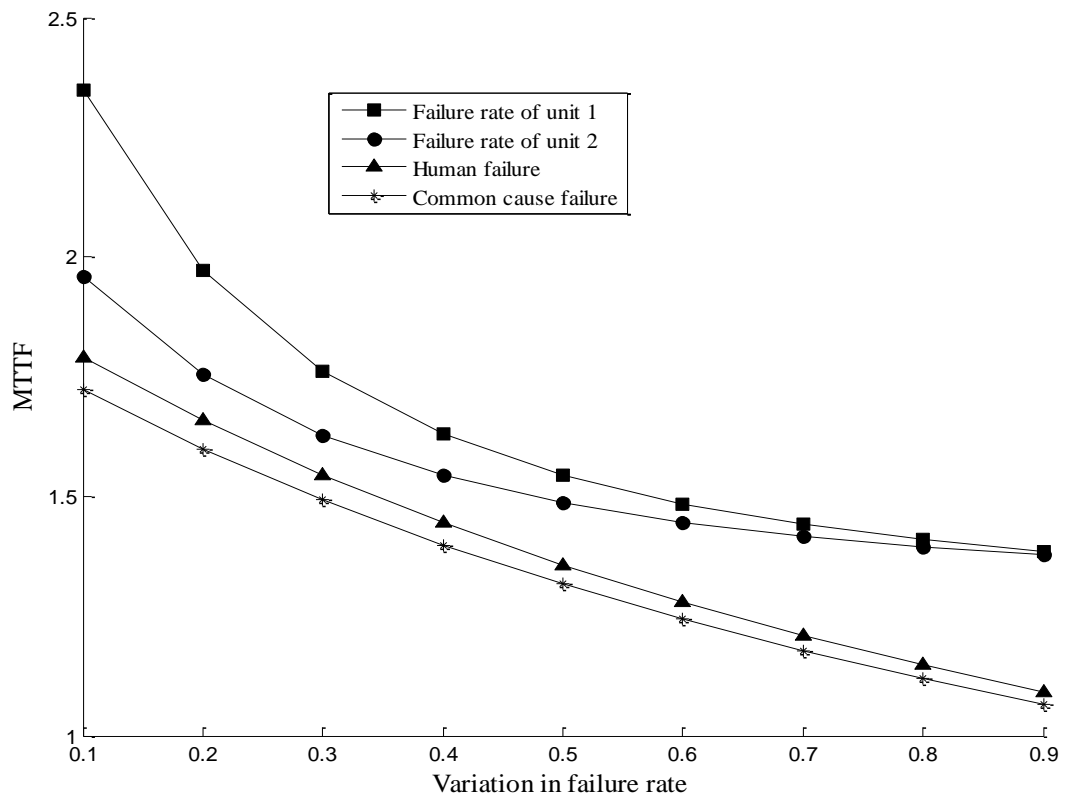

Figure 4. MTTF as function of failure rates in the Comprehensive State 
International Journal of Mathematical, Engineering and Management Sciences

Vol. 4, No. 1, 116-130, 2019

https://dx.doi.org/10.33889/IJMEMS.2019.4.1-011

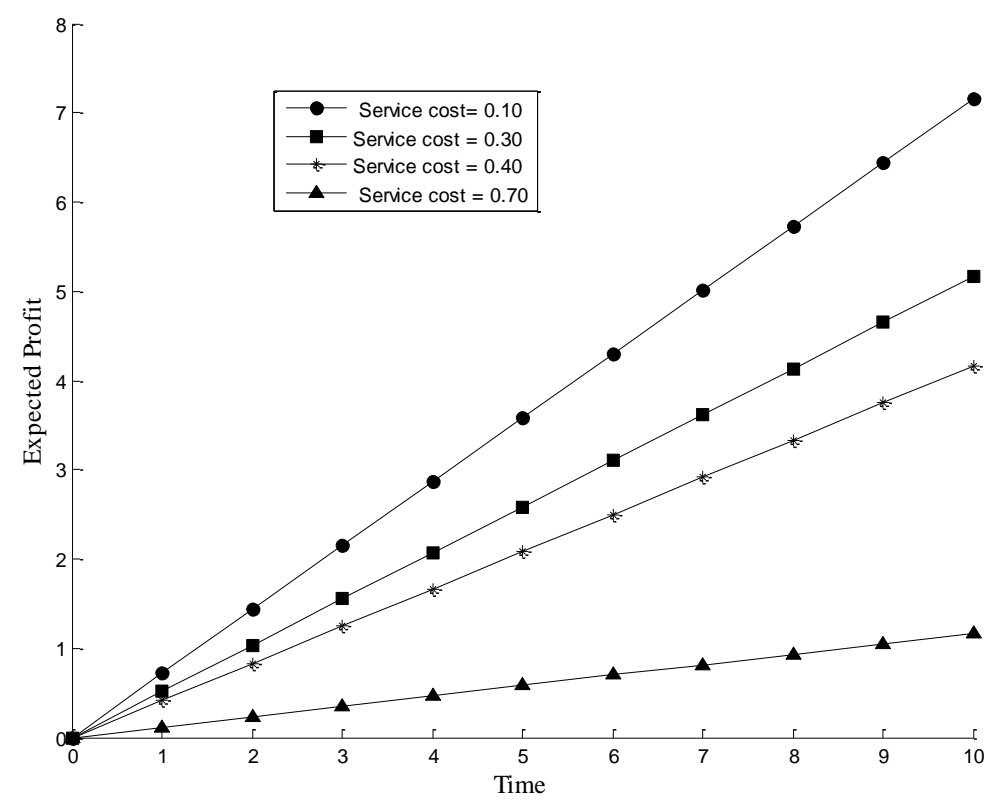

Figure 5. Expected profit as a function of time in Comprehensive State

\section{Conclusion}

The proposed model has incorporated copula to analyze the performance of two dissimilar units parallel system by considering common cause failure, human fault and hardware failure of units. The use of non-identical units in the parallel network decreases system cost and hence considered the system is more economical as compared to systems having identical units. Such dissimilar units parallel systems are widely found in power generating stations, aircrafts, medical equipments and industrial setups. The present model is significant because it has two repair facilities in completely failed states. The present system has maximum availability and reliability when there is no human error. Moreover, results reveal that the system has minimum availability and reliability in the comprehensive state. This study shows that at any moment, system availability is more than its reliability. Graph of system MTTF in comprehensive state illustrates that with an increase in values of each failure rate MTTF decreases. Through cost analysis, it is found that the rising service cost decreases the expected profit. So, the present system can be made more reliable by preventing the occurrence of the human error and common cause failure.

\section{Conflict of Interest}

The authors confirm that this article contents have no conflict of interest.

\section{Acknowledgement}

The authors would like to express their sincere thanks to the referee and for their valuable suggestions towards to the improvement of the paper. 
International Journal of Mathematical, Engineering and Management Sciences

Vol. 4, No. 1, 116-130, 2019

https://dx.doi.org/10.33889/IJMEMS.2019.4.1-011

\section{References}

Agnihotri, R. K., \& Satsangi, S. K. (1996). Two-non-identical unit-system with priority-based repair and inspection. Microelectronics Reliability, 36(2), 279-282.

Chopra, G., \& Ram, M. (2017). Stochastic analysis of two non-identical unit parallel system incorporating waiting time. International Journal of Quality and Reliability Management, 34(6), 817-832.

Chung, W. K. (1981). A two non-identical three-state units redundant system with common-cause failures and one standby unit. Microelectronics Reliability, 21(5), 707-709.

Deswal, S., \& Malik, S. C. (2015). Reliability measures of a system of two non-identical units with priority subject to whether conditions. Journal of Reliability and Statistical Studies, 8(1), 181-190.

Dhillon, B. S., \& Anuda, O. C. (1993). Common-cause failure analysis of a non-identical unit parallel system with arbitrarily distributed repair times. Microelectronics Reliability, 33(1), 87-103.

Dhillon, B. S., \& Viswanath, H. C. (1991). Reliability analysis of a non-identical unit parallel system with common-cause failures. Microelectronics Reliability, 31(2-3), 429-441.

Dhillon, B. S., \& Yang, N. (1992). Stochastic analysis of standby systems with common-cause failures and human errors. Microelectronics Reliability, 32(12), 1699-1712.

Dhillon, B. S., \& Yang, N. (1993). Availability of a man-machine system with critical and non-critical human error. Microelectronics Reliability, 33(10), 1511-1521.

EL-Sherbeny, M. S. (2013). Stochastic analysis of a two non-identical unit parallel system with different types of failures subject to preventive maintenance and repairs. Mathematical Problems in Engineering, Vol. 2013 Article ID192545, 10 pages.

Gupta, P. P., \& Agarwal, S. C. (1984). A parallel redundant complex system with two types of failure under preemptive-repeat repair discipline. Microelectronics Reliability, 24(3), 395-399.

Gupta, R., Kishan, R., \& Kumar, P. (1999). A two-non-identical-unit parallel system with correlated lifetimes. International Journal of Systems Science, 30(10), 1123-1129.

Kontoleon, J. M., \& Kontoleon, N. (1974). Reliability analysis of a system subjected to partial and catastrophic failures. IEEE Transactions on Reliability, 23(4), 277-278.

Kumar, A., Ram, M., \& Rawat, R. S. (2017). Optimization of casting process through reliability approach. International Journal of Quality and Reliability Management, 34(6), 833-848.

Kumar, P., Bharti, A., \& Gupta, A. (2012). Reliability analysis of a two non-identical unit system with repair and replacement having correlated failures and repairs. Journal of Informatics and Mathematical Sciences, 4(3), 339-350.

Li, J. (2016). Reliability comparative evaluation of active redundancy vs. standby redundancy. International Journal of Mathematical, Engineering and Management Sciences, 1(3), 122-129.

Malik, S. C., Bhardwaj, R. K., \& Grewal, A. S. (2010). Probabilistic analysis of a system of two nonidentical parallel units with priority to repair subject to inspection. Journal of Reliability and Statistical Studies, 3(1), 1-11.

Nelsen, R. B. (2006). An introduction to the Copulas (2 ${ }^{\text {nd }}$ Edition). New York: Springer-Verlag.

Ram, M. (2013). On system reliability approaches: a brief survey. International Journal of System Assurance Engineering and Management, 4(2), 101-117.

Ram, M., \& Goyal, N. (2018). Bi-directional system analysis under copula-coverage approach. Communications in Statistics-Simulation and Computation, 47(6), 1831-1844. 
International Journal of Mathematical, Engineering and Management Sciences

Vol. 4, No. 1, 116-130, 2019

https://dx.doi.org/10.33889/IJMEMS.2019.4.1-011

Ram, M., \& Singh, S. B. (2008). Availability and cost analysis of a parallel redundant complex system with two types of failure under preemptive-resume repair discipline using Gumbel-Hougaard family copula in repair. International Journal of Reliability, Quality and Safety Engineering, 15(04), 341-365.

Ram, M., \& Singh, S. B. (2010). Availability, MTTF and cost analysis of complex system under preemptive-repeat repair discipline using Gumbel-Hougaard family copula. International Journal of Quality \& Reliability Management, 27(5), 576-595.

Ram, M., Singh, S. B., \& Singh, V. V. (2013). Stochastic analysis of a standby system with waiting repair strategy. IEEE Transactions on Systems, Man and Cybernetics: Systems, 43(3), 698-707.

Saxena, A., Singh, S. K., Kumar, A., \& Ram, M. (2019). Water treatment system performance evaluation under maintenance policies. In System Performance and Management Analytics (pp. 147-165). Springer, Singapore.

Singh, V. V., \& Gulati, J. (2014). Availability and cost analysis of a standby complex system with different type of failure under waiting repair discipline using Gumbel-Hougaard copula distribution. In proceedings of International Conference on Issues and Challenges in Intelligent Computing Techniques (pp. 180-186). IEEE Publishing.

Sridharan, V., \& Mohanavadivu, P. (1997). Reliability and availability analysis for two non-identical unit parallel systems with common cause failures and human errors. Microelectronics Reliability. 37(5), 747-752.

Zheng, J., Okamura, H., \& Dohi, T. (2018). Reliability importance of components in a real-time computing system with standby redundancy schemes. International Journal of Mathematical, Engineering and Management Sciences, 3(2), 64-89. 